\title{
Next Stop: Postheteronormativität
}

\author{
Neue Reflexionsimpulse zum Thema Geschlechter-, Sexualitäts- und Beziehungsvielfalt \\ für die (offene) Kinder- und Jugendarbeit
}

Geschlechterreflektierende Soziale Arbeit bewegt sich zwischen zwei entgegengesetzten Anforderungen: Einerseits will sie aus ihrer Tradition heraus Kinder und Jugendliche bei der Navigation durch gesellschaftliche Geschlechter- und Sexualitätsnormen unterstützen, indem sie diese kennenlernen und sich ggf. daran anpassen. Andererseits soll sie die Adressat_innen im Unterlaufen dieser Normen bestärken und ihnen neue Handlungsspielräume eröffnen. Eine postheteronormative Perspektive in der Sozialen Arbeit ist vor allem auf letzteres gerichtet, indem sie die Vielfältigkeit geschlechtlicher und sexueller Entwicklungen radikal anerkennt und über heteronormative Gesellschaftsordnungen und Handlungsmuster hinausweist.

$\mathrm{M}$ it dem 2018 beschlossenen möglichen dritten Geschlechtseintrag „divers“ im Geburtenregister und der schon vorher bestehenden Option, dort kein Geschlecht einzutragen, haben sich die Möglichkeiten der Geschlechtszuordnung real erweitert. Auch die sogenannte „Ehe für alle“ ist eine rechtliche Realität. Treten wir damit schon in ein postheteronormatives Zeitalter ein? Sind also Weiblichkeit und Männlichkeit sowie Heterosexualität keine maßgeblichen Orientierungs- und Ordnungsfolien mehr, die ihre Macht dadurch festigen, dass sie andere Geschlechter und Sexualitäten als abweichend oder besonders erscheinen lassen? So einfach ist einer tief in die

\footnotetext{
Mart Busche

Alice Salomon Hochschule Berlin,

Berlin, Deutschland

*1975; Diplom-Politikwissenschaftler*in, derzeit tätig an der Alice Salomon Hochschule Berlin im BMBF-Projekt „Jungen*pädagogik und Prävention von sexualisierter Gewalt. Potenziale und Herausforderungen männlichkeitsbezogener Jugendarbeit, Sexualpädagogik, Prävention sexualisierter Gewalt sowie queerer Bildung (JupP)".

mart.busche@ash-berlin.eu
}

Zusammenfassung Die Möglichkeiten, Geschlecht, Sexualität und Beziehungen auf vielfältige Weise zu leben, erweitern sich beständig und stellen die Offene Kinder- und Jugendarbeit wie auch die Kinder- und Jugendhilfe vor alte und neue Herausforderungen. Wie kann die Arbeit möglichst integrativ und wenig ausschließend umgesetzt werden? Unter dem Stichwort der „Postheteronormativität“ bietet der Beitrag einige Reflexionsimpulse an, wie eine zeitgemäße Pädagogik sich entsprechend aufstellen und positionieren kann.

Schlüsselwörter Heteronormativität, Queer, Geschlecht, Sexualität, Diversität, Offene Kinder- und Jugendarbeit, Soziale Arbeit
Gesellschaft eingeschrieben heteronormativen Struktur wohl nicht beizukommen, aber die rechtlichen Veränderungen weisen darauf hin, dass das System der Zweigeschlechtlichkeit und Heterosexualität als Norm umkämpft sind und sich Perspektiven auf Gleichberechtigung und Antidiskriminierung - wie sie etwa im Kinder- und Jugendhilfegesetz ( $\mathbb{9}$ Abs. 3 SGB VIII) und im Allgemeinen Gleichbehandlungsgesetz $(\mathbb{S} 1 \mathrm{AGG}) \mathrm{zu}$ finden sind - um die Frage der Lebbarkeit weiterer Geschlechter- und Sexualitätsentwürfe erweitern. Welche Bedarfe sind für die Soziale Arbeit und insbesondere die Offene Kinder- und Jugendarbeit (OKJA) zu erkennen, wenn es um die vielfältigen Lebensweisen geht, in denen Kinder und Jugendliche ihr Geschlecht und ihre Sexualität ausgestalten? Den Ansatz der Pädagogik vielfältiger Lebensweisen aufgreifend und damit Erkenntnissen der poststrukturalistischen Gender und Queer Studies im Anschluss an Judith Butler folgend, wird dabei - anders als beim alltagstheoretischen Verständnis von „sexueller und geschlechtlicher Vielfalt" - eine Auseinandersetzung mit und Kritik an Heteronormativität zentral gesetzt (vgl. Hartmann 2002).

\section{Normen und Normalitäten}

Eine poststrukturalistische Perspektive nimmt die Vielheit, Widersprüchlichkeit und Wandelbarkeit (geschlechtlicher und sexueller) Bedeutungen in den Blick und macht damit auf die Brüchigkeit dominanter Ordnungen aufmerksam: Was in einem Moment als „normal“ gilt, muss es im nächsten schon nicht mehr sein. Insbesondere wenn die Herstellungsbedingungen allgemeingültig erscheinender Normalitäten offengelegt und hinterfragt werden, können weitere Realitäten als gül- 


\section{Extrablick: Kinder- und Jugendhilfe und LGBTIQ*}

tige und legitime Lebensweisen in den Blick kommen. In diesem Sinne ist ein postheteronormativer Zugang in der Sozialen Arbeit auch eine Einladung zum „postnormalistischen“ Denken (Hartmann et al. 2018b). Die Offene Kinder- und Jugendarbeit (OKJA) regelt durch die von ihr (implizit oder explizit) vertretenen Normen und Normalitäten, wie und als was jemand anerkannt, also in der jeweiligen Seinsweise verstanden und akzeptiert bzw. geduldet, wird. Sie strukturiert damit die Rahmenbedingungen, innerhalb derer Kinder und Jugendliche sich artikulieren und zeigen, also als Subjekte in Erscheinung treten können. Notwendigerweise sind solche Normen begrenzend; zugleich wirken sie ermöglichend, für die Frage, wer oder was mensch sein kann. Sie sind in ihrer Transformation träge, aber nicht unveränderlich, wie die oben genannten Gesetzesänderungen zeigen, die das Ringen um gesellschaftliche Veränderungen abbilden.

\section{Einrichtungen der OKJA als Agentinnen von Subjektivierungsprozessen}

„Durch welche Normen werde ich zwangsweise bestimmt, wenn ich frage, was ich werden kann? Und was geschieht, wenn ich etwas zu werden beginne, was im gegebenen Wahrheitsregime nicht vorgesehen ist? “ (Butler 2009, S. 98) Dies fragt die Philosophin Judith Butler im Nachdenken darüber, wie wir zu Subjekten werden in einer Welt, in die wir geworfen werden, ohne die Rahmenbedingungen mitbestimmen zu können, in der wir fundamental abhängig sind von der Fürsorge und Anerkennung anderer. Die vorherrschenden geschlechtlichen und sexuellen Normen geben dabei eine Struktur vor, in der ein bestimmtes geschlechtliches und/oder sexuelles Erscheinen näher liegt als ein von den Normen abweichendes. Subjektivierung ist ein Prozess, in dem wir innerhalb der vorgegebenen normativen Rahmenbedingungen in Erscheinung treten und von anderen dabei (an)erkannt werden. Einrichtungen der geschlechterreflektierenden Pädagogik denken in besonderer Intensität darüber nach, wie sie solche Strukturen und Normen der Anerkennung möglichst offenhalten, so dass keine Subjektivierungsweise von vorneherein ausgeschlossen wird bzw. Ausschlüsse von Subjektivierungsangeboten aus professionellen Gründen in Kauf genommen werden. Exemplarisch steht hierfür die Mädchenarbeit, die sich im Spannungsfeld von Schutzanspruch, Empowerment und Dekonstruktion der Kategorie Mädchen bewegt. In vielen Einrichtungen der offenen Mädchenarbeit wird seit Jahren die Öffnung der Kategorie Mädchen diskutiert, oft symbolisiert durch das Gendersternchen am Ende einer Geschlechtsbezeichnung ( „Mädchen“*) (vgl. LAG Mädchenpolitik NRW 2020) oder durch einen Unterstrich („Mädchen_“) (vgl. Busche et al. 2010). Damit wird signalisiert, dass die Kategorie Mädchen bedeutungsoffen ist - und dass weder der Geschlechtseintrag in der Geburtsurkunde noch ein bestimmter Körper Voraussetzung für eine Anerkennung als Mädchen Ausschlag gebend sind. Es gilt allein die Selbstdefinition: Wer sich als Mädchen positioniert, ist eins. Diese Perspektive öffnet den Blick auf die Vielfältigkeit und Eigensinnigkeit der sich so positionierenden Personen: von Orientierungen entlang klassischer, aber dennoch widersprüchlicher Weiblichkeitsinszenierungen, der Auseinandersetzung mit gleich-, gegengeschlechtlichen oder ganz anderen Begehrensweisen, die mit Geschlecht verknüpft sind, über die Begleitung von Jugendlichen, die vom weiblichen ins männliche Geschlechterspektrum wechseln. Die Erfahrungen der Fachkräfte, wie die Kategorie „Mädchen*" mit Leben gefühlt und auch herausgefordert wird, sind unabgeschlossen divers.

Ein Mädchen ist ein Mädchen* ist ein Mädchen_...? Neulich begab es sich, dass es bei einer solchen Freizeiteinrichtung für Mädchen* an der Tür klingelte und zwei Kinder im Alter von etwa neun Jahren dastanden, die aufgrund von Äußerlichkeiten von den meisten Menschen hierzulande in die Kategorie „Junge“ einsortiert werden würden. Beide gaben der diensthabenden Pädagogin zu verstehen, dass sie manchmal Mädchen sind bzw. sein wollen. Und dass sie gerne eingelassen werden wollen. ${ }^{1}$ Diese Situation wirft Fragen auf: Rechtfertigt das theoretische und praktisch vertretene Verständnis der Kategorie „Mädchen* “ das Einlassen der beiden Kinder - und wenn ja, wie weit soll die Öffnung der Kategorie betrieben werden? Kann oder soll sie sich gar auflösen? Wie reagieren die anderen Besucherinnen* auf die beiden neuen Kinder, auch vor dem Hintergrund der Erfahrung, dass Begegnungen mit trans* Männlichkeiten bei einigen durchaus für Irritationen gesorgt haben? Wie ließe sich eine Begegnung pädagogisch professionell begleiten? Mit Blick auf die mehrheitlich gemischtgeschlechtliche Organisation der OKJA ließe sich zudem fragen, was die Mädchen*einrichtung für die beiden Kinder so attraktiv macht, und was sie woanders vielleicht vermisst haben: Warum ist der Satz „Ich bin ein Mädchen “ anderswo für sie vielleicht nicht sagbar? Es gehört zum Alltag von pädagogischen Fachkräften, auf solch herausfordernde Weise unter Handlungsdruck zu geraten und Entscheidungen zu treffen. Fachkräfte nutzen dazu ihr professionelles Wissen und ihre Intuition, die als Teil der professionellen Haltung immer wieder auf ihre theoretische Fundierung und ihre Aktualität überprüft werden. Herausforderungen er- 
geben sich vor allem dort, wo Spannungsfelder auftreten, etwa zwischen dem Anspruch, Schutz zu gewähren, aber auch ein subjektivierendes Empowerment zu ermöglichen, oder dem Anspruch, „geschlechtsspezifischen " Bedarfen gerecht werden zu wollen und gleichzeitig die Existenz von etwas "Geschlechtsspezifischem" in Frage zu stellen. An der geschilderten Situation aus der Offenen Mädchen*arbeit lässt sich sehen, wie ambivalent ein männlich wahrgenommener Körper und die (situative) Selbstdefinition als Mädchen miteinander verkoppelt sind. Es bietet sich hier an, zuerst einmal von einer „Geschlechtsdiversität“ auszugehen, ohne eine Entscheidung in Richtung trans* zu fällen oder eine andere Festlegung vorzunehmen. Der Begriff bietet eine theoretisch gewollte Vagheit, die reflektiert, dass Geschlecht als vierdimensionales soziales Konstrukt auf und innerhalb der Ebenen von Geschlechtskörper, -ausdruck, Selbstdefinition („Identität“) und dem mit dem Geschlecht verknüpften (physischen und emotionalen) Begehren eine komplexe und widersprüchliche Angelegenheit ist. Dazu kommen weitere soziale Faktoren und ihre intersektionalen Dynamiken, also die Interaktionen und Verwobenheiten mit anderen Zugehörigkeits- und Differenzkategorien. Welche Professionalisierungsprozesse können die OKJA unterstützen, möglichst vielfältige und intersektional ausgerichtete Subjektivierungsangebote zu machen, die es Kindern und Jugendlichen eröffnen, mit ihren spezifischen Lebensweisen in Erscheinung zu treten und dabei anerkannt und wertgeschätzt zu werden? Solche Angebote versuchen, die oft herrschende Logik von Norm(alität) und Abweichung zu unterwandern, indem sie diese zum Ausgangspunkt einer Auseinandersetzung machen, deren Ziel die Ermutigung zur eigensinnigen Erkundung von Handlungsspielräumen jenseits dominanter Normalitätsvorstellungen ist.

\section{Die Diversität von Geschlecht und Sexualität ist schon da}

Dazu ist es hilfreich wahrzunehmen, dass sich viele Kinder und Jugendliche in Bezug auf Geschlecht und Sexualität bereits in einem geschlechts- oder sexualitätsdiversen Spektrum verorten. Dass sich etwa $35 \%$ der Jugendlichen zwischen den Polen „typisches Mädchen“ und „typischer Junge“ positionieren, ergab eine Onlinebefragung unter 12- bis 25-Jährigen aus der Jugend(verbands)arbeit und dem Sport $(N=364)(\mathrm{Bu}-$ sche et al. 2016, S. 151). Fast $15 \%$ wählten eine Position in der Mitte bzw. gaben „weder noch“ als Geschlechtszuordnung an. Knapp die Hälfte der befragten Jugendlichen ordnete sich einem der beiden Pole zu.
Daran wird deutlich, dass Kinder und Jugendliche sich zwar mehrheitlich im Bereich „Mädchen“ oder „Junge" verorten, aber insgesamt geschlechtlich vielfältig positionieren. Laut einer Studie des Deutschen Jugendinstituts zur Lebenssituation von lesbischen, schwulen, bisexuellen und trans* Jugendlichen und jungen Erwachsenen nutzen $24 \%$ der Befragten im Alter von 14 bis 27 Jahren für sich Selbstbeschreibungen jenseits binärer Definitionen wie „transgender“, „queer“ oder „genderfluid“ (vgl. Krell und Oldemeier 2015, S. 16). Weitere sechs Prozent wollen sich nicht geschlechtlich kategorisieren lassen. Die Grenzen solcher quantitativer Forschung sind, dass wir nicht wissen, wie die Kinder und Jugendlichen ihre geschlechtliche oder sexuelle Positionierung qualitativ füllen, was sich für sie hinter ,typisch Junge“ oder „weder-noch“ an Bedeutung verbirgt. Hier ist die OKJA aufgrund ihrer gesprächs- und interaktionsfreundlichen Kultur in einer besseren Position, der gelebten Bedeutung Raum zu geben. Als professionelle Aufgabe stellt sich ihr dabei neben Antidiskriminierung und Sichtbarmachung auch noch die Herausforderung, zu ergründen, wie sie daran mitwirken kann, dass Kindern und Jugendlichen ermöglicht wird, „die Bedeutung dieser Vielfalt als Gestaltungshorizont für sich selbst wie für ein wertschätzendes Miteinander in der Gesellschaft zu erkennen " (Hartmann und Busche 2018, S. 20).

\section{Heteronormative Otheringprozesse aushebeln}

Auch wenn die Tür in den meisten Jugendeinrichtungen nicht wie in dem zitierten Beispiel als Nadelöhr dient, das anhand der „richtigen“ Geschlechtszugehörigkeit passiert wird, so bedeutet dies nicht, dass Geschlecht (oder Sexualität) nicht trotzdem hinter der Tür eine ordnende Rolle hat. Daraufhin deuten die Zahlen zu Diskriminierungserfahrungen derjenigen hin, die sich jenseits dominanter Männlichkeits-, Weiblichkeits-, bzw. (Hetero)Sexualitätsvorstellungen bewegen. In einer Pilotstudie des Deutschen Jugendinstituts gaben $85 \%$ der befragten LSBTIQ+-Jugendlichen und jungen Erwachsenen an, Diskriminierung aufgrund ihrer Lebensweise erfahren zu haben (vgl. Krell 2013, S. 10). Solchen Jugendlichen fehlen die entsprechenden Vorbilder, sie werden mitunter von Scham und Selbstzweifeln ob ihres vermeintlichen Andersseins begleitet, sie haben Angst vor Ausgrenzung (vgl. Kleiner 2015, S. 34f.). Wenn geschlechtsdiverse und queere Kinder und Jugendliche also nicht sichtbar werden, dann tun sie dies aus gutem Grund: Die Möglichkeit liegt nahe, auf abwertende Weise "geothert“ zu werden, also als „Anders“ markiert zu werden. Dies geschieht entlang vorherrschender Normen, die so wirkmächtig sind, „dass diejenigen, die als 


\section{Extrablick: Kinder- und Jugendhilfe und LGBTIQ*}

Andere markiert werden, sich auch selbst in diesen Diskurs einfügen und auf Konstruktionen und Zuschreibungen zurückgreifen, die sie zu Anderen machen“ (Riegel 2016, S. 53) Nur über die Reflexion der in einer Einrichtung vorherrschenden geschlechtlichen und sexuellen Normen kann erkannt werden, wie konkrete Prozesse des Otherings funktionieren. Wie kommt es zustande, dass manche Kinder und Jugendliche sich als geschlechtlich und sexuell „anders“ inszenieren müssen, weil sie keine anderen Möglichkeiten haben, sich zu zeigen und damit verstanden zu werden - auch wenn sie damit Abwertungen riskieren? Wie kommt es, dass andere völlig unmarkiert bleiben (können)? Sollen Normen verschoben werden, kann es hilfreich sein, neue geschlechtliche und sexuelle Selbstverständlichkeiten und eine vielfaltsorientierte Wertschätzungskultur zu etablieren. Will ein pädagogisches Team auf dem Weg in ein postheteronormatives Zeitalter hier nicht nur diskriminierungssensibel, sondern auch ermöglichend wirken, dann gilt es Konzeptionen von einer Geschlechter, Sexualitäts- und Beziehungsvielfalt zu entwickeln, die diese nicht als das bunte Beiwerk zu der Norm von Cisgeschlechtlichkeit - also wenn gefühltes und bei der Geburt zugewiesenes Geschlecht übereinstimmen - , Heterosexualität und Paarbeziehung begreift, sondern auch letztgenannte ganz selbstverständlich als Teil dieser Vielfalt versteht und ihre Widersprüchlichkeit und Brüchigkeit wahrnimmt (vgl. Hartmann et al. 2018a, S. 184ff.).

\section{Postheteronormative Settings}

Postheteronormative Situationen und Räume wären solche, in denen heteronormative Verhältnisse weitgehend überwunden sind. „Hier erhalten all jene Menschen, die sich quer zu Zweigeschlechtlichkeit und zur Dichotomie von Hetero- und Homosexualität bewegen, den gleichen Raum, wie die, die sich cisgeschlechtlich und heterosexuell verstehen, ohne sich erklären oder legitimieren zu müssen. “ (Hartmann et al. 2018a, S. 184) Darin ist angedeutet, dass es um Entlastung von herrschenden Geschlechts- und Sexualitätsnormen geht, indem Erklärungen dazu ausbleiben können, aber auch um das Raumhaben für neue geschlechts- und sexualitätsbezogene Inszenierungen. Damit ist für die Fachkräfte verbunden, offen zu sein für noch unbekannte Geschlechter- und Sexualitätskonstellationen, die die bis dahin gültigen Verständnisse von Geschlechts(nicht)zugehörigkeit und (a)sexueller Begehrensweise irritieren oder unterlaufen.

Wollen Kinder und Jugendliche über für sie heikle geschlechts- oder sexualitätsbezogene Dinge reden, dann kann auf der Basis von Erkenntnissen aus der Forschung zur Prävention sexualisierter Gewalt gegen Jungen* da- von ausgegangen werden, dass Fachkräfte auf spezifische Weise einen dafür geeigneten Raum zum Reden anbieten oder herstellen (vgl. Scambor et al. 2021). Dies kann ein enttabuisierendes Sprechen sein, das ganz selbstverständlich (eigene) Erfahrungen mit Gewalt, das Wissen um verunsichernde sexualisierte Situationen oder auch die Vielfalt von Geschlechtern und Sexualitäten in pädagogische Einheiten oder auch Alltagsgespräche einflechtet. Auch Kinder und Jugendliche stellen solche Räume (mit) her, etwa in einem Pausen- oder Tür-und-Angel-Gespräch. Ein postheteronormativer Zugang würde dabei von einer real existierenden Vielfalt ausgehen und diese weiterdenken, ohne die binäre Logik von Norm und Abweichung erneut herzustellen. Die beiden Kinder aus dem Beispiel wurden übrigens in die Mädchen*einrichtung eingelassen. Damit gingen sowohl bei den bereits anwesenden Mädchen* als auch beim pädagogischen Team einige Irritationen einher: Die Fragen danach, wer oder was ein „Mädchen* “ ist, und was die Mädchen"einrichtung wem anbieten kann, wurden neu gestellt. Unterschiedliche Normalitätsannahmen werden sichtbar, die das Team nun in einem professionellen Reflexionsprozess analysiert. Pluralität und Konflikthaftigkeit der unterschiedlichen geschlechtlichen und sexuellen Realitäten konzeptionell willkommen zu heißen, bildet eine wichtige Bedingung auf dem Weg in postheteronormative pädagogische Verhältnisse.

Eingegangen. 11. Dezember 2020

Angenommen. 15. Januar 2021

Funding. Open Access funding enabled and organized by Projekt DEAL.

Open Access. Dieser Artikel wird unter der Creative Commons Namensnennung 4.0 International Lizenz veröffentlicht, welche die Nutzung, Vervielfältigung, Bearbeitung, Verbreitung und Wiedergabe in jeglichem Medium und Format erlaubt, sofern Sie den/die ursprünglichen Autor(en) und die Quelle ordnungsgemäß nennen, einen Link zur Creative Commons Lizenz beifügen und angeben, ob Änderungen vorgenommen wurden.

Die in diesem Artikel enthaltenen Bilder und sonstiges Drittmaterial unterliegen ebenfalls der genannten Creative Commons Lizenz, sofern sich aus der Abbildungslegende nichts anderes ergibt. Sofern das betreffende Material nicht unter der genannten Creative Commons Lizenz steht und die betreffende Handlung nicht nach gesetzlichen Vorschriften erlaubt ist, ist für die oben aufgeführten Weiterverwendungen des Materials die Einwilligung des jeweiligen Rechteinhabers einzuholen.

Weitere Details zur Lizenz entnehmen Sie bitte der Lizenzinformation auf http://creativecommons.org/licenses/by/4.0/deed.de 
1. Aus Anonymisierungsgründen verschweige ich weitere Details und bedanke mich sehr herzlich beim pädagogischen Team dieser Einrichtung, dass es mir das Beispiel für diesen Beitrag zur Verfügung stellt.

\section{Literatur}

Busche, M., Domann, S., Krollpfeiffer, D., Norys, T., \& Rusack, T. (2016). Perspektiven auf sexualisierte Gewalt im Kontext der Jugend(verbands)arbeit und des Jugendsports - Aspekte geschlechtlicher und sexueller Vielfalt. In C. Mahs, B. Rendtorf \& T. V. Rieske (Hrsg.), Erziehung - Gewalt - Sexualität. Zum Verhältnis von Geschlecht und Gewalt in Erziehung und Bildung (S. 147-169). Opladen: Verlag Barbara Budrich.

Busche, M., Maikowski, L., Pohlkamp, I., \& Wesemüller, E. (Hrsg.). (2010). Feministische Mädchenarbeit weiterdenken. Zur Aktualität einer bildungspolitischen Praxis. Bielefeld: transcript.

Butler, J. (2009). Die Macht der Geschlechternormen und die Grenzen des Menschlichen. Frankfurt/M.: Suhrkamp.

Hartman, J., \& Mart, B. (2018). Mehr als Sichtbarmachung und Antidiskriminierung. Perspektiven einer Pädagogik vielfältiger geschlechtlicher und sexueller Lebensweisen. Sozial Extra, 5, 20-24. https://doi.org/10.1007/ s12054-018-0073-8.

Hartmann, J. (2002). Vielfältige Lebensweisen. Dynamisierungen in der Triade Geschlecht-Sexualität-Lebensform. Kritisch-dekonstruktive Perspektiven für die Pädagogik. Opladen: Leske \& Budrich.

Hartmann, J., Busche, M., Nettke, T., \& Streib-Brzič, U. (2018a). Where to go on? Mögliche nächste Schritte im Professionalisierungsprozess. In M. Busche, J. Hartmann, T. Nettke \& U. Streib-Brzič (Hrsg.), Heteronormativitätskritische Jugendbildung. Reflexionen am Beispiel eines museumspädagogischen Modellprojekts. Bielefeld: transcript.

Hartmann, J., Köbsell, S., \& Schäuble, B. (2018b). Neue Selbstverständlichkeiten etablieren - post-normalistische Perspektiven im Studium der Sozialen Arbeit. In Freie Universität Berlin (Hrsg.), Toolbox. Gender und Diversity in der Lehre. www.genderdiversitylehre.fu-berlin.de/toolbox/_ content/pdf/Hartmann-Koebsell-Schaeuble-2018.pdf (06.01.2021).

Kleiner, B. (2015). subjekt bildung heteronormativität. Rekonstruktion schulischer Differenzerfahrungen lesbischer, schwuler, bisexueller und Trans" jugendlicher. Opladen: Verlag Barbara Budrich.

Krell, C. (2013). Abschlussbericht der Pilotstudie „Lebenssituationen und Diskriminierungserfahrungen von homosexuellen Jugendlichen in Deutschland". München: DJI. www.dji.de/fileadmin/user upload/lebenssituationen_lgbt/Abschlussbericht_Pilotstudie_Lebenssituationen_und_Diskriminierungserfahrungen_von_homosexuellen_Jugendlichen_in_Deutschland. pdf (09.12.2020)

Krell, C., \& Oldemeier, K. (2015). Coming-out-und dann ...? Ein DJIForschungsprojekt zur Lebenssituation von lesbischen, schwulen, bisexuellen und trans* Jugendlichen und jungen Erwachsenen. München: DJI. www.dii.de/fileadmin/user_upload/bibs2015/DJI_Broschuere_ComingOut. pdf (09.12.2020)

LAG Mädchenarbeit in NRW e. V. (2020). Betrifft Mädchen (2. Aufl.). Weinheim: Beltz Juventa.

Riegel, C. (2016). Bildung - Intersektionalität - Othering. Pädagogisches Handeln in widersprüchlichen Verhältnissen. Bielefeld: transcript Verlag.

Scambor, E., Täubrich, M., Busche, M., Könnecke, B., \& Hartmann, J. (2021). Potenziale einer heteronormativitätskritischen sexuellen Bildung für die Prävention sexualisierter Gewalt gegen Jungen. In schulheft 2. Wien: Verein der Förderer der Schulhefte.

Hier steht eine Anzeige.

Springer 